\title{
Efficacy of Intravenous Tranexamic Acid in Reducing Blood Loss after Elective Cesarean Section
}

\author{
Samir Abd Allah Ali, Tamer Fares Oof, Mohamed Fouad Elmolla
}

Department of Obstetrics \& Gynecology, Faculty of Medicine, Al-Azhar University, Cairo, Egypt

Corresponding author: Mohamed Fouad Elmolla, Mobile: 01279837613,email: sara234.rr@gmail.com

\begin{abstract}
Background: Primary post-partum hemorrhage (PPH) is defined as blood loss greater than or equal to $500 \mathrm{ml}$ within 24 hours after birth, while severe PPH is blood loss greater than or equal to $1000 \mathrm{ml}$ within 24 hours.

Objective: was to determine the efficacy and safety of tranexamic acid in reduction of blood loss after the elective cesarean sections.

Patients and Methods: We performed a randomized, controlled study of 200 pregnant females who underwent elective CS. The patients had attended the Labor Ward in Al-Azhar University Maternity Hospital. 100 of them received tranexamic acid 20 minutes before beginning of anesthesia in addition to oxytocin after delivery of the baby; the other 100 patients received oxytocin only.

Results: In the $1^{\text {st }}$ two hours post-partum vaginal bleeding was significantly less severe in study group than control group ( $\mathrm{p}<0.019)$. There was nonsignificant difference between study and control groups as regards preoperative hemoglobin concentration $(\mathrm{p}=0.195)$, Postoperative hemoglobin concentration was significantly greater in study group than control group ( $\mathrm{p}<0.001)$, Reduction in hemoglobin levels ware significantly less in study group than control group $(\mathrm{p}<0.001)$. There was nonsignificant difference between study and control groups as regards to preoperative hematocrite $(\mathrm{p}=0.967)$, Postoperative hematocrite levels were significantly higher in study group than control group (p0.015), Reduction in hematocrite levels ware significantly less in study group than control group $(\mathrm{p}<0.001)$.
\end{abstract}

Keywords: Cesarean section, lower segment cesarean section

\section{INTRODUCTION}

Cesarean section is defined as the delivery of a baby through a surgical abdominal incision. Cesarean section (CS) rates are increasing to as high as 25 to 30 $\%$ in many areas of the world ${ }^{(l)}$.

Delivery by CS causes more complications than normal vaginal delivery. Infection can occur at the incision site, in the uterus and in other pelvic organs such as the bladder. blood loss in a cesarean delivery is greater than with vaginal deliveries. This can increase levels of anemia or a blood transfusion (1 to 6 women delivered by CS per 100 require a blood transfusion), increase the possibility of injury to organs such as the bladder or bowel. Scar tissue may travel inside the pelvic region causing blockage and pain. Adhesions also increases levels of future pregnancy complications such as placenta previa or placental abruption. Extended hospital stays and recovery time. There may be a negative reaction to the anesthesia given during a cesarean or negative reaction to pain medication given after the operation.

The increase of the Risk of additional surgeries: Includes possibility of hysterectomy, bladder repair or another cesarean. Risks and complications may occur to the baby e.g.; premature birth breathing problems, low APGAR scores and fetal injuries may occur ${ }^{(2)}$.

One of these common complications is primary or secondary postpartum hemorrhage (20\%). It leads to the increase of maternal mortality and morbidity. Postpartum hemorrhage causes approximately $25 \%$ of worldwide maternal deaths (WHO Recommendations 2006) and also causes $12 \%$ severe anemia of survivors. In order to reduce maternal mortality and morbidity rates caused by bleeding, it is important to reduce the amount of bleeding during and after cesarean sections ${ }^{(3)}$.

In severe cases, CS may cause obstetric hemorrhage, hysterectomy, admission to an intensive care unit, or maternal death. Medications, such as oxytocin, misoprostol, prostaglandin F2 $\alpha$, and tranexamic acid, have been used to control bleeding during and after $\mathrm{CS}^{(4)}$.

Tranexamic acid is a synthetic derivative of the amino acid lysine that has an antifibrinolytic effect through the reversible blockade of the lysine binding sites on plasminogen molecules. The intravenous administration of tranexamic acid has been routinely used for many years to reduce hemorrhage during and after surgical procedures like coronary artery bypass, oral surgery, orthotopic liver transplantation, total hip or knee arthroplasty, and urinary tract surgery ${ }^{(5)}$.

Tranexamic acid is very useful in reducing blood loss and incidence of blood transfusion in the surgeries. Tranexamic acid treatment is inexpensive and would be considered highly cost effective in high, middle- and low-income countries ${ }^{(6)}$.

\section{AIM OF THE WORK}


The aim of the current study was to determine the efficacy and safety of tranexamic acid in reduction of blood loss after the elective cesarean sections.

\section{PATIENTS AND METHODS}

This randomized, controlled study included a total of 200 pregnant females who underwent elective CS, attending at the Labor Ward in Al-Azhar University Maternity Hospital. Approval of the ethical committee and a written informed consent from all the subjects were obtained. This study was conducted between May 2018 to December 2018.

All women fulfilled the inclusion criteria were enrolled in the study. The patients were randomized into 2 groups using a computer-generated randomization list, generated using medcalc $\odot$ version 13(medcalc@ software, mariakirke, ostend, Belgium).

Group (A): received $1 \mathrm{~g}$ of intravenous tranexamic acid in $200 \mathrm{~mL}$ of normal saline (study group; $\mathrm{n}=100$ ). Group (B): received no the tranexamic acid (control group; $\mathrm{n}=100)$.

The randomization list was concealed and accessed by sequentially numbered, opaque, sealed envelopes (SNOSE); immediately before intervention. The solution was prepared by an anesthetist who was not involved in patient's management or assessment.

Inclusion criteria: pregnant women undergoing cesarean delivery for many elective indications. Full term primiparas / multiparas. Singleton pregnancy being delivered by CS.

Exclusion criteria: Medical problems involving the heart, liver, kidney and brain diseases. Blood disorders. Allergy to tranexamic acid. History of thromboembolic disorders, abnormal placentation, severe pre-eclampsia, uterine anomalies and pathology. Multiple pregnancy, macrosomia. Polyhydramnios. Patients requiring blood transfusion due to anemia

All Patients were subjected to completed history taking and clinical examination and preoperative investigations (complete blood count, prothrombin time, activated partial thromboplastin time, liver and kidney function tests).

In the study group patients. 20 minutes before taking the skin incision $1 \mathrm{~g}$ tranexamic acid (Kapron ${ }^{\circledR}$, Amoun, Egypt) was given in $200 \mathrm{~mL}$ normal saline. After delivery of the neonate, 5 units of oxytocin (Syntocynon ${ }^{\circledR}$, NOVARTIS, Egypt) in $500 \mathrm{ml}$ normal saline was given by intravenous drip over 30 minutes.

Tranexamic acid injections were prepared by diluting $1 \mathrm{~g}(10 \mathrm{~mL})$ tranexamic acid with $200 \mathrm{ml}$ of normal saline.

Tranexamic acid was not given in the control group patients. After delivery of the neonate, oxytocin was given as in the study group patents.

The blood loss was measured at 2 periods. following placental delivery to the end of the surgery (1st period), and from the end of the operation to 2 hours after birth(2nd period).

Uterine contractility, placental separation, neonatal manifestations, and side effects caused by tranexamic acid ware noted as nausea, vomiting ,diarrhea ,thrombosis ,blurring of vision and hypotension.

\section{Measuring blood loss}

The amount of blood loss $(\mathrm{mL})=$ (weight of the used towels in every period - weight of the towels prior to the surgeries) + the volume sucked in the suction bottle after placental delivery in $\mathrm{mL}$. In addition, the pads used after completion of CS to two hours postpartum were separately assessed. Hemoglobin, hematocrit values were noted 24 hours after operation for both groups. Conversion of weight of towels by $(\mathrm{g})$ to volume by $(\mathrm{ml})$ by equation $(1000$ $\mathrm{g}=962 \mathrm{ml}$ ). Heart rates, respiratory rates and blood pressure were checked and noted before the surgery, immediately after placental delivery and one and two hours after birth, respectively.

Primary outcome measures: Amount of blood loss assessed during cesarean section (in $\mathrm{ml}$ ) after placental delivery till end of the operation.

Secondary outcome measures: Vital data during first two post operative hours. Vaginal bleeding were assessed during first two post-operative hours. 24 hours post operative hemoglobin and hematocrit. Maternal and neonatal side effects of tranexamic acid.

Sample Size Justification Sample size was calculated using data from previous studies ${ }^{(7)}$, data from Cochrane systematic review that showed the risk of post-partum blood loss $>400 \mathrm{~mL}$ was $14.44 \%$ in pregnant women who received tranexamic acid (TXA), in contrast to $32.38 \%$ in women who were not ${ }^{(8)}$ and EpiInfo version 7.0, setting the power at $80 \%$, the two-sided confidence level at $95 \%$ and $10 \%$ patients drop rates. Calculation according to these values, the minimal number of women needed to produce a statistically acceptable figure were 98 in each group. Therefore, two hundred (200) were recruited at the beginning of the current study to be randomized into two groups.

\section{Statistical analysis}

Data were analyzed using Statistical Program for Social Science (SPSS) version 18.0. Quantitative data were expressed as mean \pm standard deviation (SD). Qualitative data were expressed as frequency and percentage.

The following tests were done: Independentsamples t-test of significance was used when comparing between two means. Chi-square (X2) test of significance was used in order to compare proportions between two qualitative parameters. Probability (P-value) - P-value $<0.05$ was considered significant. $-\mathrm{P}$-value $<0.001$ was considered as highly significant. - P-value $>0.05$ was considered insignificant. 


\section{RESULTS}

Table (1): Descriptive data of the group (A) study group and group (B) control group.

This table should be table number (1)

\begin{tabular}{|l|r|r|r|r|r|r|r|r|}
\hline & \multicolumn{4}{|c|}{ Group (A) Study group } & \multicolumn{4}{c|}{ Group (B) Control group } \\
\hline \multicolumn{1}{|c|}{ Descriptive data } & Min. & Max. & Mean & \pm SD & Min. & Max. & Mean & \pm SD \\
\hline Age (years) & 18 & 39 & 27.81 & 5.07 & 19 & 40 & 28.32 & 4.65 \\
\hline BMI (kg/m ${ }^{2}$ ) & 23 & 35.8 & 29.24 & 3 & 16.7 & 35 & 29.55 & 3.08 \\
\hline GA (weeks) & 37 & 40 & 38.19 & 0.7 & 37 & 41 & 38.22 & 1.1 \\
\hline Systolic BP (preoperative) & 100 & 140 & 117.84 & 9.4 & 100 & 130 & 117.63 & 7.98 \\
\hline Diastolic BP (preoperative) & 60 & 90 & 74.54 & 6.19 & 60 & 80 & 75 & 5.29 \\
\hline Systolic BP (postoperative) & 100 & 130 & 114.59 & 6.01 & 100 & 120 & 109.74 & 8.16 \\
\hline $\begin{array}{l}\text { Diastolic BP } \\
\text { (postoperative) }\end{array}$ & 60 & 80 & 74.66 & 5.75 & 60 & 88 & 70.63 & 7.48 \\
\hline HR (BPM) (preoperative) & 65 & 110 & 85.01 & 10.18 & 65 & 110 & 85.8 & 8.7 \\
\hline HR (BPM) (postoperative) & 70 & 105 & 83.14 & 7.21 & 75 & 120 & 96.12 & 10.66 \\
\hline Hb. (g/dl) (preoperative) & 9.8 & 15 & 12.16 & 1.28 & 9.5 & 15 & 11.9 & 1.24 \\
\hline Hb. (g/dl) (postoperative) & 9.2 & 14 & 11.44 & 1.24 & 8.1 & 14.2 & 10.55 & 1.18 \\
\hline HCT\% (preoperative) & 30 & 45 & 37.07 & 3.54 & 27 & 49 & 37.1 & 4.13 \\
\hline HCT\% (postoperative) & 25 & 43 & 34.47 & 3.74 & 23 & 42 & 32.91 & 3.97 \\
\hline Blood loss 1st period (ml) & 180 & 840 & 386.55 & 104.98 & 220 & 850 & 507.76 & 152.14 \\
\hline Blood loss 2nd period (ml) & 10 & 270 & 114.73 & 53.82 & 10 & 320 & 139.67 & 73.35 \\
\hline Total blood loss (ml) & 220 & 1030 & 501.28 & 119.2 & 295 & 1140 & 647.43 & 178.77 \\
\hline
\end{tabular}

Table (2): Comparison between group A and group B as regard demographic data.

\begin{tabular}{|c|c|c|c|c|c|c|}
\hline Demographic data & \multicolumn{2}{|c|}{ Group (A) } & & \multicolumn{2}{|c|}{ Group (B) } & t-test \\
\hline Mean & $\pm \mathrm{SD}$ & Mean & & $\pm \mathrm{SD}$ & $\mathrm{t}$ & $\mathrm{p}$-value \\
\hline Women Age (years) & 27.81 & 5.07 & 28.32 & 4.65 & -0.636 & 0.526 \\
\hline $\mathrm{BMI}(\mathrm{kg} / \mathrm{m} 2)$ & 29.24 & 3.00 & 29.55 & 3.08 & -0.624 & 0.533 \\
\hline GA (weeks) & 38.19 & 0.70 & 38.22 & 1.10 & -0.228 & 0.820 \\
\hline Duration of 1.S C.S(min) & 42.65 & 8.57 & 43.28 & 21.87 & 0.268 & 0.788 \\
\hline
\end{tabular}

This table shows no statistically significant difference between groups as regard demographic data, using Independent sample t-test with p-value >0.05 NS

Table (3): Comparison between group A and group B as regard parity.

\begin{tabular}{|c|c|c|c|c|}
\hline \multirow{2}{*}{ Parity } & \multicolumn{2}{|c|}{ Group (A) } & \multicolumn{2}{c|}{ Group (B) } \\
\cline { 2 - 5 } & No. & \% & No. & \% \\
\hline P0 & 22 & 22.0 & 14 & 14.0 \\
\hline P1 & 32 & 32.0 & 29 & 29.0 \\
\hline P2 & 31 & 31.0 & 37 & 37.0 \\
\hline P3 & 4 & 4.0 & 7 & 7.0 \\
\hline PG & 11 & 11.0 & 13 & 13.0 \\
\hline Total & 100 & 100.0 & 100 & \\
\hline x2 & \multicolumn{3}{|c}{3.299} \\
\hline p-value & \multicolumn{3}{|c|}{0.0} \\
\hline
\end{tabular}

This table shows no statistically significant difference between groups as regard parity using Chi-square test with pvalue $>0.05 \mathrm{NS}$ 
Table (4): Comparison between group A and group B as regard blood pressure and heart rate.

\begin{tabular}{|c|c|c|c|c|c|c|}
\hline \multirow{2}{*}{ Blood pressure } & \multicolumn{2}{|c|}{ Group (A) } & \multicolumn{2}{c|}{ Group (B) } & \multicolumn{2}{c|}{ t-test } \\
\cline { 2 - 7 } & Mean & $\mathbf{\pm S D}$ & Mean & $\mathbf{\pm S D}$ & t & p-value \\
\hline Systolic BP (preoperative) & 117.84 & 9.40 & 117.63 & 7.98 & 0.145 & 0.885 \\
\hline Diastolic BP (preoperative) & 74.54 & 6.19 & 75.00 & 5.29 & -0.490 & 0.625 \\
\hline Systolic BP (2hrs postoperative) & 114.59 & 6.01 & 109.74 & 8.16 & 4.142 & $<\mathbf{0 . 0 0 1}$ \\
\hline Diastolic BP (2hrs postoperative) & 74.66 & 5.75 & 70.63 & 7.48 & 3.693 & $<\mathbf{0 . 0 0 1}$ \\
\hline HR (BPM) (preoperative) & 85.01 & 10.18 & 85.80 & 8.70 & -0.511 & 0.610 \\
\hline HR (BPM) (2hrs postoperative) & 83.14 & 7.21 & 96.12 & 10.66 & -8.712 & $<\mathbf{0 . 0 0 1}$ \\
\hline
\end{tabular}

This table shows statistically significant difference between groups as regard systolic and diastolic Blood pressure postoperative, using Independent sample t-test with p-value $<0.001 \mathrm{HS}$, the rest have insignificant. Also, statistically significant difference between groups as regard heart rate postoperative.

Table (5): Comparison between group A and group B as regard Hemoglobin (Hb) and Hematocrite(HCT\%).

\begin{tabular}{|l|c|c|c|c|c|c|}
\hline \multirow{2}{*}{} & \multicolumn{2}{|c|}{ Group (A) } & \multicolumn{2}{|c|}{ Group (B) } & \multicolumn{2}{c|}{ t-test } \\
\cline { 2 - 7 } & Mean & $\mathbf{\pm S D}$ & Mean & $\mathbf{\pm S D}$ & $\mathbf{t}$ & p-value \\
\hline Hb. level (g/dl) (preoperative) & 12.16 & 1.28 & 11.90 & 1.24 & 1.302 & 0.195 \\
\hline Hb. Level (g/dl) (24hrs postoperative) & 11.44 & 1.24 & 10.55 & 1.18 & 4.511 & $<\mathbf{0 . 0 0 1 ( H S )}$ \\
\hline HCT\% level (preoperative) & 37.07 & 3.54 & 37.10 & 4.13 & -0.041 & 0.967 \\
\hline HCT\% level (24hrs postoperative) & 34.47 & 3.74 & 32.91 & 3.97 & 2.470 & $\mathbf{0 . 0 1 5}(\mathbf{S})$ \\
\hline
\end{tabular}

This table shows highly statistically significant difference between groups as regard $\mathrm{Hb}$. (g/dl) postoperative, using Independent sample t-test with p-value $<0.001 \mathrm{HS}$, while $\mathrm{Hb}$. (g/dl) preoperative non-significant, also statistically significant difference between groups as regard HCT\% postoperative.

Table (6): Comparison between group A and group B as regard blood loss.

\begin{tabular}{|l|l|l|l|l|l|l|}
\hline & \multicolumn{2}{|c|}{ Group (A) } & \multicolumn{2}{l|}{ Group (B) } & \multicolumn{2}{l|}{ t-test } \\
\hline & Mean & \pm SD & Mean & $\mathbf{\pm S D}$ & T & p-value \\
\hline $\begin{array}{l}\text { Blood loss in 1st period (ml) } \\
\text { (from placental delivery till end of operation) }\end{array}$ & 386.55 & 104.98 & 507.76 & 152.14 & -5.665 & $<\mathbf{0 . 0 0 1 ( H S )}$ \\
\hline $\begin{array}{l}\text { Blood loss in 2nd period (ml) } \\
\text { (from end of operation till 2 hrs } \\
\text { postoperative) }\end{array}$ & 114.73 & 53.82 & 139.67 & 73.35 & -2.369 & $\mathbf{0 . 0 1 9}(\mathbf{S})$ \\
\hline Total blood loss (ml) & & & & & & \\
\hline
\end{tabular}

This table shows statistically significant difference between groups as regard blood loss $1^{\text {st }}$ and $2^{\text {nd }}$ periods and total blood loss with difference [146.15 ml (22.6\%)] blood loss in group (A) less than group (B), using Independent sample t-test.

Table (7): Comparison between group A and group B as regard intraoperative events.

\begin{tabular}{|l|c|c|c|c|}
\hline \multirow{2}{*}{ Intraoperative events } & \multicolumn{2}{c|}{ Number of cases } & \multicolumn{2}{c|}{ Chi-square } \\
\cline { 2 - 5 } & Group (A) & Group (B) & X2 & P-value \\
\hline Accessory hemostatic Sutures of the uterine incision & 33 & 38 & 0.546 & 0.460 \\
\hline Uterine artery injury or ligation & 2 & 3 & 0.205 & 0.651 \\
\hline Broad ligament hematoma & 0 & 0 & 0.000 & 1.000 \\
\hline Blood transfusion & 0 & 1 & 1.005 & 0.316 \\
\hline Hysterectomy & 0 & 0 & 0.000 & 1.000 \\
\hline
\end{tabular}

This table shows no statistically significant difference between groups as regard intraoperative events, using Chi-square test, with p-value $>0.05 \mathrm{NS}$ 
Table (8): Differences between preoperative and postoperative data as regard all parameters in group (A).

\begin{tabular}{|c|c|c|c|c|c|c|}
\hline \multirow{2}{*}{ Parameters } & \multicolumn{2}{|c|}{ Group (A) } & \multicolumn{2}{|c|}{ Paired Difference } & \multicolumn{2}{|c|}{$\begin{array}{c}\text { Paired Sample t- } \\
\text { test }\end{array}$} \\
\hline & Mean & $\pm \mathrm{SD}$ & Mean & $\pm \mathrm{SD}$ & $\mathrm{T}$ & p-value \\
\hline Systolic Bpr (preoperative) & 117.84 & 9.40 & \multirow{2}{*}{3.24} & \multirow{2}{*}{10.08} & \multirow{2}{*}{2.77} & \multirow{2}{*}{$\begin{array}{c}0.007 \\
(\mathrm{~S})\end{array}$} \\
\hline Systolic Bpr (2hrs postoperative) & 114.59 & 6.01 & & & & \\
\hline Diastolic Bpr (preoperative) & 74.54 & 6.19 & \multirow{2}{*}{-0.12} & \multirow{2}{*}{8.07} & \multirow{2}{*}{1.08} & \multirow{2}{*}{0.896} \\
\hline Diastolic $\mathrm{Bpr}$ (2hrs postoperative) & 74.66 & 5.75 & & & & \\
\hline $\mathrm{HR}(\mathrm{BPm})$ (preoperative) & 85.01 & 10.18 & \multirow{2}{*}{1.88} & \multirow{2}{*}{9.07} & \multirow{2}{*}{-0.76} & \multirow{2}{*}{0.079} \\
\hline $\mathrm{HR}(\mathrm{BPm})$ (2hrs postoperative) & 83.14 & 7.21 & & & & \\
\hline $\mathrm{Hb} .(\mathrm{g} / \mathrm{dl})$ (preoperative) & 12.16 & 1.28 & \multirow{2}{*}{0.73} & \multirow{2}{*}{0.58} & \multirow{2}{*}{10.77} & \multirow{2}{*}{$\begin{array}{c}<0.001 \\
\text { (HS) }\end{array}$} \\
\hline $\mathrm{Hb}$. (g/dl) (24hrs postoperative) & 11.44 & 1.24 & & & & \\
\hline HCT\% (preoperative) & 37.07 & 3.54 & \multirow{2}{*}{2.61} & \multirow{2}{*}{2.26} & \multirow{2}{*}{9.94} & \multirow{2}{*}{$\begin{array}{l}<0.001 \\
\text { (HS) }\end{array}$} \\
\hline HCT\% (24hrs postoperative) & 34.47 & 3.74 & & & & \\
\hline Blood loss 1st period (ml) & 386.55 & 104.98 & \multirow{2}{*}{271.82} & \multirow{2}{*}{116.73} & \multirow{2}{*}{20.03} & \multirow{2}{*}{$\begin{array}{c}<0.001 \\
\text { (HS) }\end{array}$} \\
\hline Blood loss 2nd period (ml) & 114.73 & 53.82 & & & & \\
\hline
\end{tabular}

This table shows statistically significant difference between preoperative and postoperative as regard systolic Blood pressure, Hemoglobin, HCT\% and blood loss, the rest have insignificant, using Independent sample t-test, in group (A).

Table (9): Differences between preoperative and postoperative as regard all parameters data in group (B).

\begin{tabular}{|c|c|c|c|c|c|c|}
\hline \multirow{2}{*}{ Parameters } & \multicolumn{2}{|c|}{ Group (B) } & \multicolumn{2}{|c|}{ Paired Difference } & \multicolumn{2}{|c|}{ Paired Sample t-test } \\
\hline & Mean & $\pm \mathrm{SD}$ & Mean & $\pm \mathrm{SD}$ & $t$ & p-value \\
\hline Systolic Bpr (preoperative) & 117.63 & 7.98 & \multirow{2}{*}{7.89} & \multirow{2}{*}{9.84} & \multirow{2}{*}{6.99} & \multirow{2}{*}{$<0.001$ (HS) } \\
\hline Systolic Bpr (2hrs postoperative) & 109.74 & 8.16 & & & & \\
\hline Diastolic Bpr (preoperative) & 75.00 & 5.29 & \multirow{2}{*}{4.37} & \multirow{2}{*}{8.18} & \multirow{2}{*}{4.658} & \multirow{2}{*}{$<0.001$ (HS) } \\
\hline Diastolic Bpr (2hrs postoperative) & 70.63 & 7.48 & & & & \\
\hline $\mathrm{HR}(\mathrm{BPm})$ (preoperative) & 85.80 & 8.70 & \multirow{2}{*}{-10.32} & \multirow{2}{*}{10.17} & \multirow{2}{*}{-8.84} & \multirow{2}{*}{$<0.001$ (HS) } \\
\hline HR (BPm) (2hrs postoperative) & 96.12 & 10.66 & & & & \\
\hline $\mathrm{Hb} .(\mathrm{g} / \mathrm{dl})$ (preoperative) & 11.90 & 1.24 & \multirow{2}{*}{1.35} & \multirow{2}{*}{0.56} & \multirow{2}{*}{21.21} & \multirow{2}{*}{$<0.001$ (HS) } \\
\hline $\mathrm{Hb}$. (g/dl) (24hrs postoperative) & 10.55 & 1.18 & & & & \\
\hline HCT\% (preoperative) & 37.10 & 4.13 & \multirow{2}{*}{4.19} & \multirow{2}{*}{2.51} & \multirow{2}{*}{14.53} & \multirow{2}{*}{$<0.001$ (HS) } \\
\hline HCT\% (24hrs postoperative) & 32.91 & 3.97 & & & & \\
\hline Blood loss 1st period (ml) & 507.76 & 152.14 & \multirow{2}{*}{368.09} & \multirow{2}{*}{158.41} & \multirow{2}{*}{20.26} & \multirow{2}{*}{$<0.001$ (HS) } \\
\hline Blood loss 2nd period (ml) & 139.67 & 73.35 & & & & \\
\hline
\end{tabular}

This table shows statistically significant difference between preoperative and postoperative as regard all parameters, using Independent sample t-test, with p-value $<0.001 \mathrm{HS}$ in group (B).

Comparison between table (11) and table (12) shows: Decrease of haemoglobin in group A is less than group B. Decrease of blood pressure (systolic and diastolic) in group A is less than group B. Decrease in hematocrite in group A is less than group B. Amount of blood loss in group A is less than gro 
randomized clinical trial. J Matern Fetal Neonatal Med., 26 (1):1705-1709.

\section{REFERENCES}

1.Kambo I, Bedi N and Dhillon BS (2003): A critical appraisal of cesarean section rates at teaching hospitals in India. Int. J Gyneacol Obstet., 79:151158.

2.americanpregnancy.org/

3.Abou Zahr C (2003): Global burden of maternal death and disability. Br Med Bull., 67:1-11.

4.Güngördük K, Asicioglu O, Celikkol O , Olgac Y and Ark C (2010): Use of additional oxytocin to reduce blood loss at elective caesarean section: a randomised control trial. Aust $\mathrm{N}$ Z J Obstet Gynaecol., 50:36- 39.

5.Ido K, Neo M, Asada Y, et al. (2000): Reduction of blood loss using tranexamic acid in total knee and hip arthroplasties. Arch Orthop Trauma Surg., 120:518520.

6. Guerriero C, Cairns J, Perel P, Shakur H, Roberts I (2011): Cost-effectiveness analysis of administering tranexamic acid to bleeding trauma patients using evidence from the CRASH-2 trial. PLoS One, 6(5):e18987.

7.Abdel-Aleem H, Alhusaini TK, Abdel-Aleem MA, Menoufy $M$ and Gülmezoglu AM (2013): Effectiveness of tranexamic acid on blood loss in patients undergoing elective cesarean section:
8. Novikova $\mathbf{N}$ and Hofmeyr GJ (2010): Tranexamic acid for preventing postpartum haemorrhage. https://www.ncbi.nlm.nih.gov/pubmed/20614466

9.https://en.wikipedia.org/wiki/Compendium_of_Phar maceuticals_and_Specialties

10. Gai MY, Wu LF, Su QF, Tatsumoto K (2005): Clinical observation of blood loss reduced by tranexamic acid during and after caesarian section: a multi- center, randomized trial. European Journal of Obstetrics \& Gynecology and Reproductive Biology, 112:154-7.

11. Mayur G, Purvi P, Ashoo G, Pankaj D (2008): Efficacy of tranexamic acid in decreasing blood loss during and after cesarean section: a randomized case controlled prospective study. J Obstet Gynecol India, 57(3):227-30.

12. Yang H, Zheng S, Shi C (2004): Clinical study on the efficacy of tranexamic acid in reducing postpartum blood lose.Chinese Journal of Obstetrics and Gynaecology, 36(10):590-2.

13. Sekhavat L, Tabatabaii A, Dalili M, Farajkhoda T, Tafti AD (2009): Efficacy of tranexamic acid in reducing blood loss after cesarean section. The Journal of Maternal-Fetal \& Neonatal Medicine, 22(1):72-5. 